\title{
Validity and User Experience in an Augmented Reality Virtual Tooth Identification Test
}

\author{
Hera Kim-Berman, Elisabeta Karl, Jason Sherbel, Lauren Sytek, Vidya Ramaswamy
}

Abstract: By leveraging emerging technologies in augmented reality (AR) and virtual reality (VR), a Virtual Dental Library and AR virtual tooth identification test were developed at a U.S. dental school. The AR virtual tooth identification test is a visionbased AR application that uses three-dimensional models of extracted human teeth as test items. The aims of this study were to investigate the validity of the AR virtual tooth identification test and evaluate the users' experience with the virtual testing method. The AR virtual tooth identification test scores were compared with real tooth identification tests, scores on three quizzes, final exam, and final grade for the course to assess its validity. In addition, a survey was used to assess students' perceptions of the AR tool. In 2018, all 109 first-year dental students who had completed the dental anatomy course were invited to participate in the study. Of the 93 participants, 61 (56\% of total students) were included in the correlation analysis ( 32 were excluded due to incomplete test answer sheets or missing criterion measures). All 93 could respond to the survey and provide comments. In the results, the AR virtual tooth identification test had a positive correlation with the real tooth identification test $(\mathrm{r}=0.410, \mathrm{p}<0.01)$, a combined score of two real tooth identification tests $(r=0.545, \mathrm{p}<0.01)$, the final exam $(r=0.489, \mathrm{p}<0.01)$, and overall grade for the dental anatomy course $(\mathrm{r}=0.661, \mathrm{p}<0.01)$. On the tests, the students had some difficulty in viewing and manipulating the images and experienced technical difficulties related to their smartphones, and their survey responses expressed little support for the AR tool. Nevertheless, this study demonstrated criterion validity of the AR virtual assessment tool for tooth identification.

Hera Kim-Berman is Clinical Assistant Professor and Graduate Orthodontic Program Director, Department of Orthodontics and Pediatric Dentistry, University of Michigan School of Dentistry; Elisabeta Karl is Clinical Assistant Professor, Department of Cariology, Restorative Sciences, and Endodontics, University of Michigan School of Dentistry; Jason Sherbel is a graduate orthodontic resident, University of Michigan School of Dentistry; Lauren Sytek is a graduate orthodontic resident, University of Michigan School of Dentistry; and Vidya Ramaswamy is Associate Director for Curriculum and Program Evaluation, University of Michigan School of Dentistry. Direct correspondence to Dr. Hera Kim-Berman, Department of Orthodontics and Pediatric Dentistry, University of Michigan School of Dentistry, 1011 N. University Dr., Ann Arbor, MI 48109-1382; 734-764-1080; bermanh@umich.edu.

Keywords: predoctoral dental education, educational technology, computer-assisted instruction, computer simulation, augmented reality, virtual reality, tooth anatomy

Submitted for publication 3/29/19; accepted 6/25/19; first published online 8/12/19

doi: $10.21815 / J D E .019 .139$

$\mathrm{D}$ ental education is based on student mastery of three areas: cognitive knowledge and critical thinking; affective domain (e.g., empathy, communication, and behavior management); and psychomotor skills required for technical procedures and treatments. Preclinical courses are used to help dental students develop fine motor skills, control of new tools, and knowledge of therapeutics, biomaterials, and techniques prior to patient care where the integration of all three domains occurs. ${ }^{1}$ As a foundational course in preclinical dental curricula, dental anatomy introduces students to the anatomical and morphological characteristics of the human dentition.

Dental anatomy is an important and integral part of dentistry since it develops spatial cognition through the study of anatomical structures' shape, location, and associations in occlusal function. Additionally, the ability to identify and recognize tooth morphology is the basis for dentists to restore lost structure and regain function. The traditional method of teaching dental anatomy combines lectures with waxing assessments that use textbooks, samples of preserved and/or manufactured teeth, and wax blocks to sculpt tooth forms. ${ }^{2,3}$ A deficiency of this method is the lack of variety of specimens, which may hamper development of students' ability to discern among ideal, clinically acceptable, and clinically unacceptable tooth morphology. ${ }^{3}$ Technology has been used to improve preclinical and clinical teaching, share educational content and methods, change the balance of power between faculty and students, and create a learner-centered environment. ${ }^{4}$ An example is a computer-based software to aid students in learning dental anatomy. ${ }^{5}$ Such software programs make use of text, photographic images, illustrations, lectures, aural pronunciation, terminology, and tests. ${ }^{6}$ 
Recently, at the University of Michigan School of Dentistry, the Virtual Dental Library, a virtual library of the human dentition, was developed by leveraging emerging technologies in augmented reality (AR) and virtual reality (VR). The Virtual Dental Library can be accessed by students using a VR head-mounted device or mobile application on smartphones to view the library contents. VR immerses users in an entirely computer-generated virtual world that replaces the real world, ${ }^{7}$ while AR supplements the real world with virtual content to enhance user perceptions. ${ }^{8}$

The Virtual Dental Library and its VR/AR applications were developed using a conceptual model of the educational triangle involving student, teacher, and learning tool and their interaction to influence academic and affective outcomes (Figure 1). The interrelationship of the educational triangle can also lead to curricular reform and iterative development of learning and assessment tools. In this model, the Virtual Dental Library is an educational tool that consists of ideal resin teeth, extracted human teeth, prepared resin teeth for operative and prosthodontic restorations, impressions, and provisional restorations. The library can be expanded and customized for each individual user: users can upload their own content or three-dimensional (3D) models using multiple file types such as FBX, OBJ, STL, WRL, PDB, DAE, and DICOM. The Virtual Dental Library contents can be accessed anywhere and at any time with advanced visualization functions and mobile learning that is interactive, engaging, and studentdriven. Users can create groups and invite peers to share 3D models from their customized libraries, and teachers can create and share virtual assessment tools such as a virtual tooth identification test.

A tooth identification test, using examples of extracted human teeth, is one of the measures often used to evaluate students' cognitive skills in dental morphology. A large room or lab space is required to conduct a tooth identification test, so that students can rotate through the different tooth stations to complete the examination. Often the set-up of the test is cumbersome and time-consuming for the faculty, and student review of the test items is challenging since many students must wait to view a single example of the test item. Sometimes the exam process is interrupted if there is a problem with the test items (e.g., a tooth is dropped or broken) and an alternate test item must be used.

To improve our current method of tooth identification testing, faculty members developed a virtual

\section{Conceptual Model}

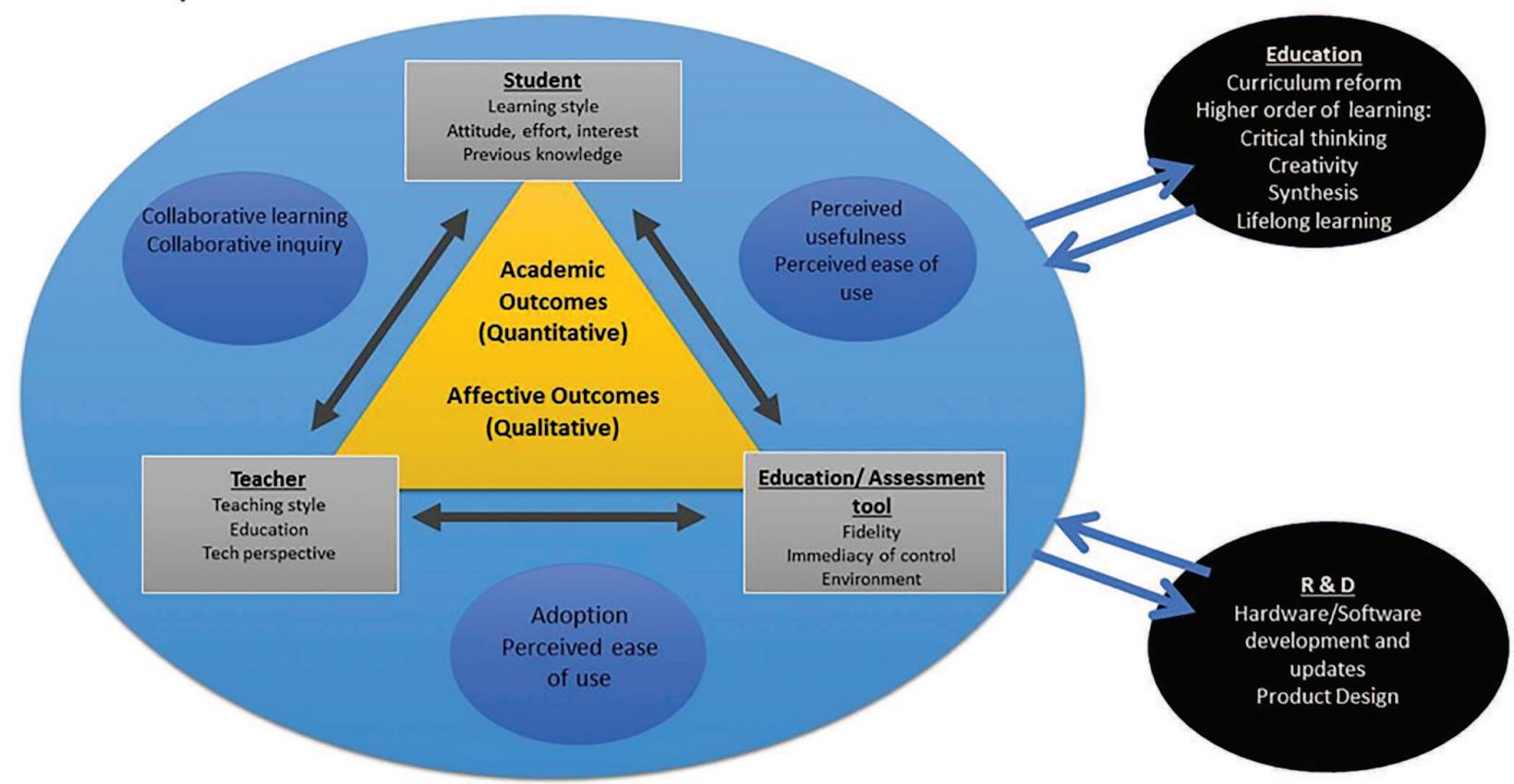

Figure 1. Virtual reality and augmented reality in dental education conceptual model 
tooth identification test using the Arthea VR engine software (Gwydion Inc., Ann Arbor, MI, USA) and the Virtual Dental Library. The AR virtual tooth identification test is a vision-based AR application that uses an AR Tag, a fiduciary marker system, to estimate the camera position and orientation, which allows for video input to gain knowledge of the environment for viewpoint tracking and virtual object interaction. ${ }^{9-12}$ The AR virtual tooth identification test consists of 3D models of extracted teeth used in previous real tooth identification tests, which were uploaded using Arthea software so that the test items can be downloaded and viewed using smartphones with an AR Tag (Figure 2).

When educational programs change an exam format (e.g., written test vs. computer-based or virtual test), the context effect in testing and the validity of the test should be assessed. ${ }^{13}$ Context effect refers to the interaction of the examinee, the test item, and the testing environment that may affect the validity of the test. The validity of an exam would allow for interpretation and provide meaningful and appropriate inferences of the test scores to student performance. For high-stakes standardized exams in dentistry such as the Dental Admission Test and the National Board Dental Examination, validity study is conducted on a yearly basis to examine test scores, student performance, and outcomes. ${ }^{13-17}$ Evidence supporting a change in test format should consist of correlation of scores from the new method of testing with criterion measures that demonstrate a correlation superior to the traditional testing method using the same measures. We found no published studies that investigated the validity of an AR assessment instrument in dentistry. The aims of this study were therefore to investigate the validity of the AR virtual tooth identification test and evaluate the users' experience with the virtual testing method.

\section{Methods}

The Health Sciences and Behavioral Sciences Institutional Review Board (IRB) at the University of Michigan determined that this study was exempt from IRB oversight (\#HUM00149081). In 2018, all 109 first-year dental students who had completed the dental anatomy course were invited to participate in the study. Students were given extra credit in the preclinical dental anatomy continuation course (in the following fall semester) for participating.

An introduction to use of the mobile application was given on two occasions: one week prior to the test and immediately before the test. The students were given access to the Virtual Dental Library to view and study dental morphology using ideal resin permanent teeth. The students did not have access to the test items prior to the exam. On the day of

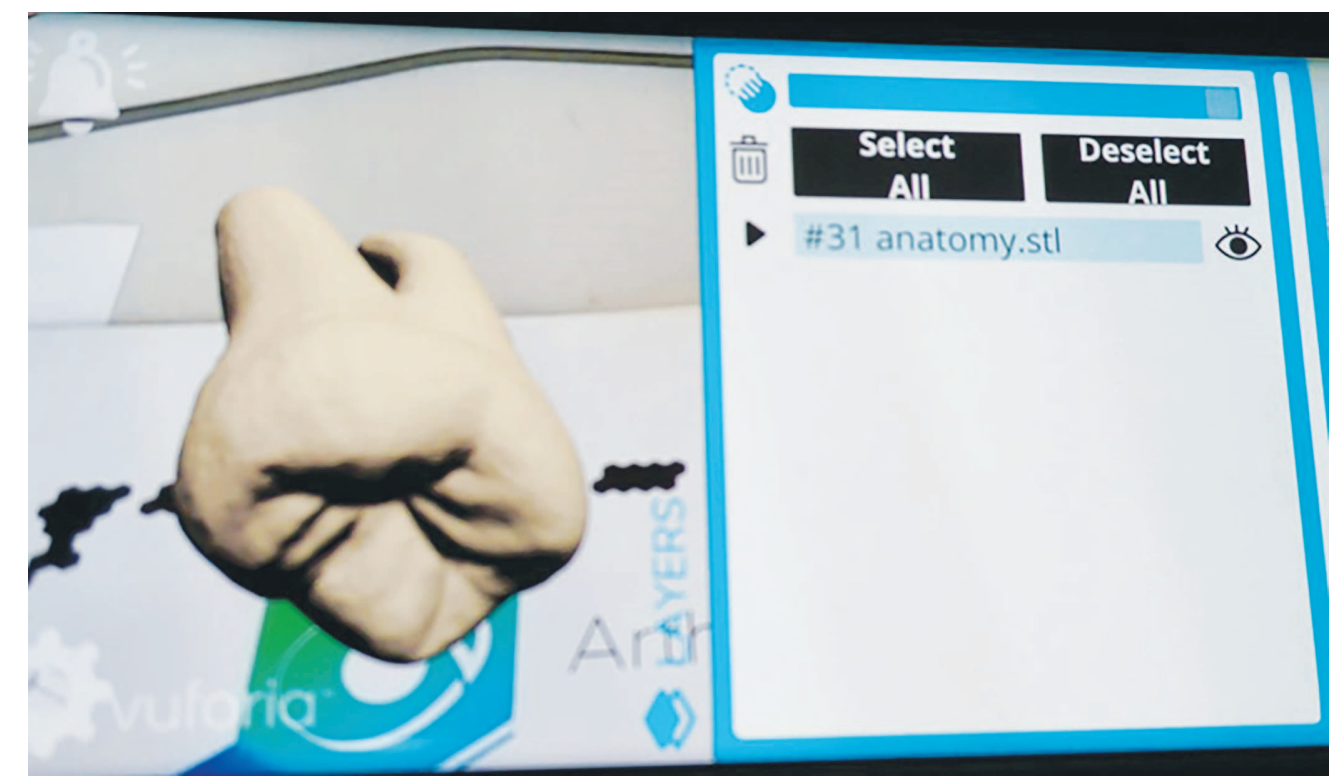

Figure 2. Example of lower right second molar (tooth \#31) from Virtual Dental Library using Arthea software and Android mobile device, Galaxy S8+, in augmented reality 
the test, the students were asked to open the Arthea application on their personal smartphones, and they then downloaded, identified, and recorded the tooth number on the provided test answer sheet. The 25 test items were shared one at a time, and the students were asked to identify each test item within a determined time frame. All test sheets were collected and scored. All 25 test questions had to be completed for the students' data to be included for analysis; if there were any missing answers, the test answer sheet was excluded from the analysis.

To determine validity, Pearson productmoment correlation coefficients were computed for the real tooth identification test and the virtual tooth identification test to evaluate the relationship among various criterion measures at the 0.05 level of significance. The criterion measures were student assessments during the dental anatomy course: a real tooth identification test ( 25 test items), a second real tooth identification test (50 questions), combined scores on three quizzes, a final comprehensive exam with 40 multiple-choice questions, and the final grade for the dental anatomy course. In the first real tooth identification test, students were asked to identify the tooth considering either its maxillary or mandibular arches and left or right position in the mouth. In the second real tooth identification test, students were asked to identify the tooth as previously assessed and particular anatomical landmarks of that tooth. Finally, the quizzes combined both types of questions without handling the teeth, and the students selected whether statements were true or false. For example, two of the statements were "In the maxillary canines, the mesial cusp ridge is longer than the distal cusp ridge" and "On lateral incisors (maxillary), the mesial outline is flatter than the distal outline (more rounded)." If any of the criterion measures were missing, the student's test answer sheet was excluded from the study.

Following the AR tooth identification test, students were asked to complete an anonymous survey
(Qualtrics, Seattle, WA, USA). On the survey, participants were asked if they agreed, disagreed, or were neutral regarding three statements: "I did better using real teeth for the tooth ID exam"; "Augmented reality tool for the tooth ID exam was easy to use"; and "Using virtual teeth accurately tests my knowledge and ability to identify the teeth on a test." One openended question asked for any comments and suggestions about how the AR tool could be improved. We included this opportunity for free responses, so students could elaborate on their agreement or disagreement. Two coders compiled and organized the free response data using the following coding process. The first coder generated the categories for coding and shared them with the second coder. After the first round of coding independently, the two coders met to resolve differences in coding and modification of coding categories. The target agreement between the two coders was $85 \%$. Additionally, user login data including number of users and duration of the average session were collected over five days prior to and on the day of the test. User login data did not include individual user information.

\section{Results}

Ninety-three of the 109 students participated $(85 \%)$. Of those 93 , only $61(67 \%)$ were included in the correlation analysis; 30 were excluded due to incomplete test answer sheets, and two were excluded due to one or more missing criterion measure. All 93 participants were given the opportunity to provide responses to the survey anonymously.

The analysis showed that the AR virtual tooth identification test and the real tooth identification test correlated with all but one of the criterion measures (Table 1). The AR virtual tooth identification test had a positive correlation with the real tooth identification test $(\mathrm{r}=0.410, \mathrm{p}<0.01)$, a combined score

Table 1. Correlations among real tooth identification (ID) test, augmented reality (AR) virtual tooth ID test, and criterion measures

\begin{tabular}{|c|c|c|c|c|c|c|}
\hline Test & $\begin{array}{l}\text { Real Tooth } \\
\text { ID Test }\end{array}$ & $\begin{array}{l}\text { AR Virtual } \\
\text { Tooth ID Test }\end{array}$ & Final Exam & $\begin{array}{l}\text { Combined } \\
\text { Quizzes }\end{array}$ & $\begin{array}{l}\text { Combined Real } \\
\text { Tooth ID Tests }\end{array}$ & $\begin{array}{l}\text { Course } \\
\text { Grade }\end{array}$ \\
\hline Real tooth ID test & 1 & $0.410^{* *}$ & $0.254^{*}$ & -0.036 & $0.665^{* *}$ & $0.538^{* *}$ \\
\hline AR virtual tooth & $0.410^{* *}$ & 1 & $0.489 * *$ & 0.243 & $0.545^{* *}$ & $0.661^{* *}$ \\
\hline
\end{tabular}

ID test

Note: Correlations were determined by Pearson product-moment correlation coefficients. Criterion measures were real tooth ID test, combined scores on two real tooth ID tests, final exam, combined scores on three quizzes, and overall grade for course. 
on the two real tooth identification tests $(\mathrm{r}=0.545$, $\mathrm{p}<0.01)$, the final exam $(\mathrm{r}=0.489, \mathrm{p}<0.01)$, and overall grade for the course $(r=0.661, p<0.01)$. The real tooth identification test had a positive correlation with the virtual tooth identification test $(r=0.410$, $\mathrm{p}<0.01$ ), a combined score on the two real tooth identification tests $(\mathrm{r}=0.665, \mathrm{p}<0.01)$, the final exam $(r=0.254, p<0.05)$, and overall grade for the course $(\mathrm{r}=0.538, \mathrm{p}<0.01)$. Neither the real nor the AR virtual tooth identification exams correlated with scores on the quizzes.

The response rates on survey questions 1,2 , and 3 were $65 \%, 62 \%$, and $52 \%$, respectively. The majority (72\%) agreed that they did better using real than virtual teeth for the tooth ID exam (Figure 3). When asked whether the AR tool was easy to use, $50 \%$ disagreed. When asked if the virtual teeth accurately tested the user's knowledge of the teeth on the test, $42 \%$ disagreed.

The free response data from the survey were organized using a coding process with two coders. ${ }^{18}$ With the study's coding procedure, each response could potentially be assigned multiple codes. In most cases, the lack of agreement was in the dissimilarity of one or two categories by one of the coders. For example, a statement coded by one coder as "visualization" and "sensory" was coded by the second coder as "visualization" and "technically difficult." Interrater agreement was calculated by counting the number of times there was total agreement on how the responses were coded. For instance, if there was partial agreement, meaning that if coders agreed on one of two codes assigned to a single response but differed on the second code, it did not count as agreement. Initial agreement between the coders ranged from $66 \%$ to $77 \%$; target agreement $(85 \%)$ was achieved in the second round of coding and ranged from $86 \%$ to $88 \%$. Any remaining disagreements were resolved after mutual discussion. Table 2 shows the top categories of codes, the number of times the code was used, description of the codes, and illustrative comments on survey questions 1,2 , and 3. The top categories of the codes were issues with viewing, manipulation, time involved with the
Q1. I did better using real teeth for the tooth ID exam.

Q2. Augmented reality tool for the tooth ID exam was easy to use.

Q3. Using virtual teeth accurately tests my knowledge and ability to identify the teeth on a test.
Disagree, $16 \%$

Neutral, $12 \%$

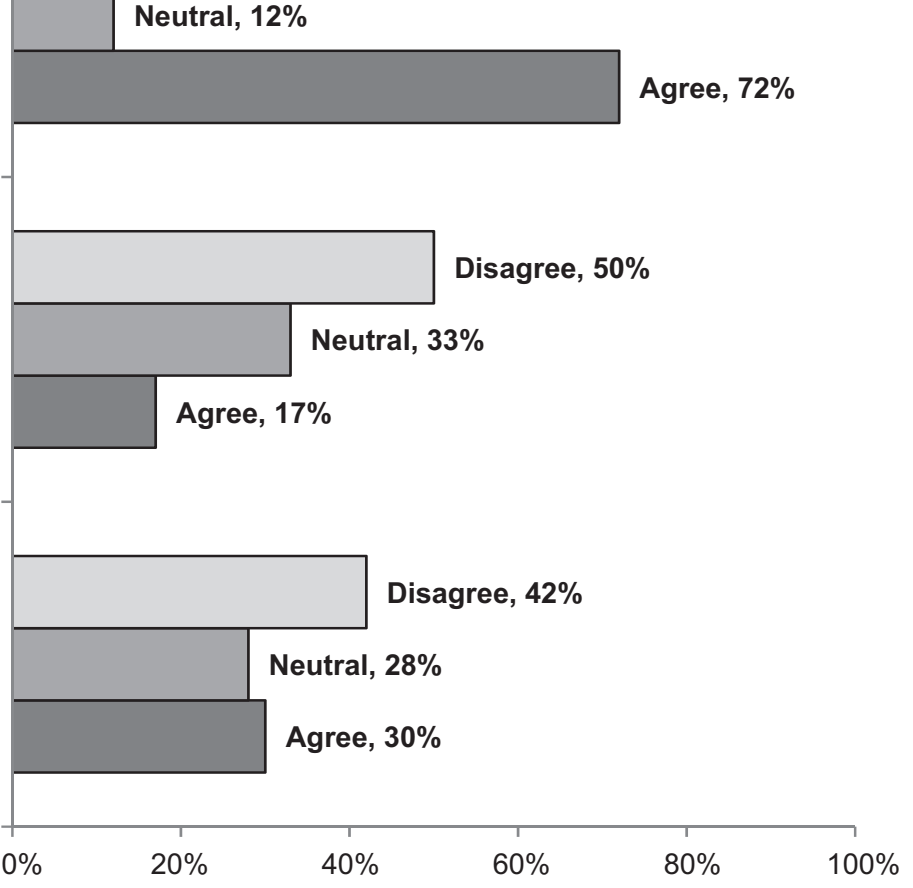

Figure 3. Responses on survey questions 1, 2, and 3

Note: Number of respondents and response rate for each question were as follows: Question 1, n=60, 65\%; Question 2, $\mathrm{n}=58,62 \%$; and Question 3, $\mathrm{n}=48,52 \%$. 
Table 2. Categorization of comments on survey questions 1, 2, and 3 regarding virtual reality (VR) and augmented
reality (AR) by top categories of codes, number of times code was used, description of code, and illustrative comments

$\begin{array}{lcl}\begin{array}{l}\text { Question, Category } \\ \begin{array}{l}\text { Question 1 } \\ \text { Viewing }\end{array} \\ \text { Manipulation }\end{array} & 192 & \begin{array}{l}\text { Description } \\ \text { The real tooth was easier to } \\ \text { see; VR made it difficult to } \\ \text { see the tooth. } \\ \text { It was difficult to move the } \\ \text { teeth in AR. }\end{array} \\ \begin{array}{l}\text { Time issues with } \\ \text { technology }\end{array} & 18 & \begin{array}{l}\text { Application took a long time } \\ \text { loading and to operate. }\end{array}\end{array}$

Question 2

Time issues with 33 Application took a long time technology loading and to operate.

"I prefer to see the real teeth in actual 3D dimension."

"I felt it was very hard to see certain features in the AVMR."

"It is easier to see fine details using the real tooth and it is much easier to manipulate."

"The teeth on the AR were not as easy to see and not as easy to quickly manipulate and move around to look for all defining features."

Time issues with loading and to operate.

"Glitchy app wouldn't load files, took forever to view whole tooth, poor scanning quality."

"It was confusing opening and closing windows, and I was losing time."

"Took too long. Easier to just have the tooth in my hand."

"I wouldn't say it was hard, but the time it took to load and the fact that you have to navigate to Files and THEN layers kind of took too much time."

"The AR tool ... was not ideal because it did not load the questions efficiently."

$\begin{array}{lcl}\text { Ease of using AR } & 27 & \begin{array}{l}\text { AR was either easy or } \\ \text { difficult to use; about 1/3 } \\ \text { thought it was difficult. }\end{array} \\ \text { Manipulation } & 18 & \begin{array}{l}\text { It was difficult to move the } \\ \text { teeth in AR. }\end{array} \\ \text { Viewing } & 12 & \begin{array}{l}\text { Tooth was difficult to see } \\ \text { against the background. }\end{array} \\ \text { Battery } & 12 & \begin{array}{l}\text { Battery drained when app } \\ \text { was used. }\end{array}\end{array}$

"Simple to use once explained."

"It really wasn't as easy to use as I had hoped. It took so long to get certain teeth to load [that] I wasn't able to even see the file before it was taken off the list."

"Rotating and manipulating the tooth on screen was difficult to adapt to, but perhaps more practice with the software would be beneficial."

"Difficult to see on the white paper background."

"It was difficult to see characteristics and features of the teeth, especially due to all surfaces being the exact same color."

"The app overheated our phones and quickly killed our batteries."

Question 3

Accuracy

24 About half thought it was not accurate, and half thought it was inaccurate.

"I do not think this performance reflects my knowledge of dental anatomy; I was more confident with my answers when I was tested using real teeth."

"The virtual teeth tested my knowledge and ability to identify teeth."

Viewing

24 The real tooth was easier to see; VR made it difficult to see the tooth. "The lack of color and poor contrast (white teeth on the white tag paper) made it difficult to distinguish some details."

"Real teeth are easier to compare size, shape, color, etc."

Note: The survey questions (with number of responses and response rate) were as follows: Question 1, "I did better using real teeth for the tooth ID exam" ( $\mathrm{n}=60,65 \%)$; Question 2, "Augmented reality tool for the tooth ID exam was easy to use" ( $\mathrm{n}=58,62 \%)$; and Question 3, "Using virtual teeth accurately tests my knowledge and ability to identify the teeth on a test" ( $=48,52 \%$ ). Only categories with more than ten codes are shown.

technology, ease of use, phone battery, and perceptions of accuracy with the AR tooth identification test.

\section{Discussion}

Thus far, only a limited number of studies have investigated the use of stereoscopy with anaglyph images in oral and maxillofacial radiography, augmented reality for operative dentistry, and virtual reality for jaw surgery simulation. ${ }^{19-23}$ Our study aimed to investigate the validity of an AR virtual tooth identification test and user experience of the testing method. A validity study is important and necessary to support changes to the exam format and content. Validity refers to accumulation of the gathered evidence from multiple sources to support the claim that the assessment is indeed evaluating the specific knowledge and skills being tested. ${ }^{24}$ 
When a new method of testing is contemplated, a validity study to examine format, content, context effects, and ease of use should be conducted prior to implementation. ${ }^{13}$

The results of this study found moderate correlation of the AR virtual tooth identification test and the traditional real tooth identification test with other criterion measures used to determine student performance in the dental anatomy course. The AR virtual tooth identification test was able to show that students' cognitive knowledge that can be interpreted from the results of real tooth identification tests, a final comprehensive exam, and the final grade for the course can also be interpreted from the results of the AR virtual tooth identification test. Correlation with the comprehensive final exam was stronger for the AR tooth identification test $(\mathrm{r}=0.489)$ than the real tooth identification test $(\mathrm{r}=0.254)$. Although the reasons for differences in the correlation are not clearly understood, it is possible that it may be related to the similarity in testing format of the AR virtual tooth identification test to the final exam. Nevertheless, there appears to be evidence to suggest that the scores on the AR virtual tooth identification test had a correlation at least equal to the traditional real tooth identification test using the same criterion measures. Although this was an exploratory study involving a short-term intervention that was implemented in a single course, given that effect sizes in educational research tend to be small, these findings are still practically relevant and may support a recommendation for change in exam format based on the validity of the AR virtual tooth identification test. One potential advantage of the AR virtual tooth identification test is efficiency. Once created, the test can be given to a number of students without the physical need for testing space and on multiple occasions. Using extracted human teeth as test items and having students rotate through tooth identification stations can be difficult to organize and coordinate and be time-consuming for the faculty. With the AR application, students can also have access to examples of previous test items for self-study and review at any time. With the traditional testing method using real teeth, student review of test items can be challenging due to limited access to a single example.

This study also evaluated students' experience with the AR virtual tooth identification test. Visionbased AR interface environments are dependent on marker implementation, calibration, type of user interface, and effective viewing and manipulation. ${ }^{9}$ The free responses indicated that the users had some difficulty in viewing and manipulating the AR application using their smartphones. Additionally, due to technical difficulties including prolonged loading time and loss of battery life on phones, there was a sample attrition rate of approximately $30 \%$. Prolonged loading time of the test items, which was partly due to inadequate bandwidth and multiple users' trying to access the network simultaneously, proved to be problematic for conducting the test. For students to be able to load the test item and have adequate time to respond, additional time to load had to be incorporated into the test structure and format. Even though extra time to load was given during the test session, many students were unable to answer the questions and complete the test. The technological problems with bandwidth, software, and phones diluted the AR intervention experience. We recommend that any future study on AR simulation address these issues with preliminary testing of the software.

Another potential reason for unfavorable user experience is that the students were unfamiliar with the AR application even though they were provided with instructions on how to use the application on two occasions (one week prior to and immediately before the AR tooth identification test). When we evaluated the user login data, there were 87 user login sessions with an average duration of seven minutes over the five days prior to the exam. Just one hour prior to the test, there were 71 user login sessions with an average duration of 49 minutes. It appears that many students spent the majority of the time getting familiar with the application immediately before the test, which may have affected their ability to become comfortable and confident using the application and new testing mode. Finally, although students reported feeling that the Virtual Dental Library can be an effective study tool, they were concerned that the variety of phone models used may give an advantage to those students with newer and better phones; this concern can create additional stress and the perception of an unequal "playing field" during a high-stakes test situation.

This study had several limitations. Technical problems with the AR virtual tooth identification test created delays during the implementation and led to $30 \%$ of the participants not completing the test. User satisfaction requires intuitive interaction that supports natural 3D object manipulation of the AR system. Although we found the AR virtual tooth identification test may be a valid part of overall assessment in dental anatomy, an iteration of the current AR application is currently being developed to improve and resolve viewing, manipulation, and 
technical issues before the next virtual tooth identification test. The results may also have been affected by students' unfamiliarity with this technology. Prior to virtual tooth identification tests in the future, multiple practice assignments should be given to ensure that students are familiar with the new technology and modality of testing. Finally, the results were based on a single cohort of volunteer students at one dental school, limiting the generalizability of the findings. Our study needs to be replicated with additional cohorts and across multiple settings.

\section{Conclusion}

Research and development in VR and AR technology as well as the growing VR and AR industry focus in the educational sector have centered on applications, educational content, and mobile learning using smartphones, collaboration, and 3D spatial interactions and experiences. Challenges remain for VR and AR in educational practices, illustrated by the technical problems encountered in our study and the limited support among these students for the AR tool. Nevertheless, the correlational analysis supported the validity of the AR virtual assessment tool for tooth identification. With a continued iterative process of user feedback and improvements with the VR and AR hardware and software, learning and assessment tools such as the Virtual Dental Library and the virtual tooth identification test may become more effective, efficient, user-friendly, and valid tools for use in dental education.

\section{REFERENCES}

1. Segura C, Halabi D, Navarro N. Design and validation of a basic dental psychomotor skills test for novice dental students. J Dent Educ 2018;82(10):1098-104.

2. De Azevedo R, Da Rosa W, Da Silva A, et al. Comparative effectiveness of dental anatomy carving pedagogy: a systematic review. J Dent Educ 2015;79(8):914-21.

3. Obrez A, Briggs C, Buckman J, et al. Teaching clinically relevant dental anatomy in the dental curriculum: description and assessment of an innovative module. J Dent Educ 2011;75(6):797-804.

4. Schleyer TK, Thyvalikakath TP, Spalleck H, et al. From information technology to informatics: the information revolution in dental education. J Dent Educ 2012;76(1):142-53.

5. Bogacki RE, Best A, Abbey LM. Equivalence study of a dental anatomy computer-assisted learning program. J Dent Educ 2004;68(8):867-71.
6. Wright E, Hendricson W. Evaluation of a 3-D interactive tooth atlas by dental students in dental anatomy and endodontic courses. J Dent Educ 2010;74(2):110-22.

7. Orlosky J, Kiyokawa K, Takemura H. Virtual and augmented reality on the $5 \mathrm{G}$ highway. J Inf Process 2017;25:133-41.

8. Bergig O, Hagbi N, El-Sana J, et al. In-place augmented reality. Virtual Reality 2011;15:201-12.

9. Liarokapis F. An augmented reality interface for visualizing and interacting with virtual content. Virtual Reality 2007;11:23-43.

10. Carlson KJ, Gagnon DJ. Augmented reality integrated simulation education in health care. Clin Sim Nurs 2016;12:123-7.

11. Lee M, Billinghurst M, Baek W, et al. A usability study of multimodal input in an augmented reality environment. Virtual Reality 2013;17:293-305.

12. Azuma RT. A survey of augmented reality. Presence Teleoperators Virtual Environ 1997;6(4):355-85.

13. Yang CL, Neumann LM, Kramer GA. Assessing context effects on test validity of the National Board Dental Examination Part I. J Dent Educ 2012;76(4):395-406.

14. Tsai TH. Validating use of a critical thinking test for the Dental Admission Test. J Dent Educ 2014;78(4):552-7.

15. Sandow PL, Jones AC, Peek CW, et al. Correlation of admission criteria with dental school performance and attrition. J Dent Educ 2002;66(3):385-92.

16. DeBall S, Sullivan K, Horine J, et al. The relationship of performance on the Dental Admission Test and performance on Part I of the National Board Dental Examination. J Dent Educ 2002;66(4):478-84.

17. Holmes DC, Doering JV, Spector M. Association among predental credentials and measures of dental school achievement. J Dent Educ 2007;72(2):142-52.

18. Payne G, Payne J. Coding qualitative data. London: Sage Publications, 2011.

19. Xia J, Samman N, Yeung R, et al. Three dimensional virtual reality surgical planning and simulation workbench for orthognathic surgery. Int J Adult Ortho Orthog Surg 2000;15(4):265-82.

20. Espejo-Trung LC, Elian SN, Luz M. Development and application of a new learning object for teaching operative dentistry using augmented reality. J Dent Educ 2015;79(11):1356-62.

21. UM3D Lab, Duderstadt Center. Learning jaw surgery with virtual reality. At: um3d.dc.umich.edu/learning-jawsurgery-with-virtual-reality/. Accessed 27 Dec. 2018.

22. Murakami S, Verdonschot RG, Kreiborg S, et al. Stereoscopy in dental education: an investigation. J Dent Educ 2017;81(4):450-7.

23. Llena C, Folguera S, Forner L, Rodríguez-Lozano FJ. Implementation of augmented reality in operative dentistry learning. Eur J Dent Educ 2018;22:e122-30.

24. Gadbury-Amyot CC, McCracken MS, Woldt JL, Brennan RL. Validity and reliability of portfolio assessment of student competence in two dental school populations: a four-year study. J Dent Educ 2014;78(5):657-67. 